\title{
A minimal supersymmetric left-right model, dark matter and signals at the LHC
}

\author{
Katri Huitu ${ }^{\mathrm{a}}$ \\ Department of Physics and Helsinki Institute of Physics, University of Helsinki, \\ P.O. Box 64 (Gustaf Hällströmin katu 2), 00014 University of Helsinki, Finland
}

Received 12 March 2020 / Accepted 30 September 2020

Published online 14 December 2020

\begin{abstract}
The left-right symmetric models extend the gauge group of the Standard Model enabling treatment of the left- and right-handed fermions in the same footing. The left-right symmetry requires the existence of right-handed neutrinos, leading naturally to non-zero masses for neutrinos. Here some aspects of a supersymmetric version of the leftright symmetric models are reviewed. Such models have many virtues, including possibility for dark matter without any new additional symmetry needed in order to have a stable lightest supersymmetric particle. In the model the lightest sneutrino or the lightest neutralino can form dark matter of the universe, at the same time fulfilling all the experimental constraints. The dark matter particle in the model can be very different from the dark matter typical in the minimal supersymmetric standard model. Specific signals for this kind of models at the LHC are also discussed.
\end{abstract}

\section{Introduction}

It is well known that the Standard Model (SM) has to be modified at some scale. Although SM is in good agreement with experiments, neutrino masses are measured to be different from zero by the oscillation of neutrino flavours, and existence of dark matter seems to be a proven fact. Other, aesthetically displeasing features, include fine-tuning of the Higgs boson mass as well as strong CP violation. One can also wonder, among other things, why there are exactly three generations, why the masses of particles differ hugely from each other, why the SM violates parity maximally.

The left-right symmetric extensions [1,2] of the Standard Model were constructed to address the last of these questions. The left-right symmetric models extend the gauge group of the Standard Model to $S U(2)_{L} \times S U(2)_{R} \times U(1)_{B-L}$ enabling treatment of the left- and right-handed fermions in the same footing. Neutrino masses can be explained by the left-right symmetric models due to the existence of the righthanded neutrinos, which are necessary in the model. The Majorana mass terms of the right-handed neutrinos will be generated in the spontaneous breaking of $S U(2)_{R}$, if it is broken by triplets. The Majorana mass term of the right-handed neutrinos can

a e-mail: katri.huitu@helsinki.f 
be large compared to the Majorana mass term of the left-handed neutrinos, and the observed tiny mass of the neutrinos can be explained by the seesaw mechanism [3-7].

The supersymmetric version of the left-right symmetric model (LRSUSY) is especially attractive from the point of view of dark matter. Contrary to the minimal supersymmetric standard model (MSSM), no new additional symmetry is needed to have a stable lightest supersymmetric particle (LSP), since R-parity is conserved due to the gauge symmetry, and if triplets with quantum number $B-L= \pm 2$ break the $S U(2)_{R}$, the R-parity remains exact also after the symmetry breaking.

Another virtue of the LRSUSY model is that it can solve both the SUSY CP problem and the strong CP problem by the parity invariance, which leads to Hermitian Yukawa-type couplings and real mass terms [8-10].

It turns out that at the tree-level of the minimal version of the model, the vacuum does not conserve the electric charge if only the neutral Higgs fields generate vacuum expectation values. It is possible to conserve charge at the tree-level, if the right-sneutrino generates a vacuum expectation value [11], or if non-renormalizable terms are included [12-14]. Minimization of the tree-level scalar potential shows that either a right-sneutrino field generates a vacuum expectation value, or electric charge conservation is lost [11]. Since the electric charge is considered to be an exact symmetry in Nature, the R-parity seems to be spontaneously broken. Such a model may well be viable, as the R-parity breaking concerns only the leptonic sector, but it will not provide a dark matter candidate, which is a disadvantage of the model. However, it was realized in reference [15] that taking into account the radiative corrections, the resulting contributions can be large enough to change the scalar potential to R-parity and charge conserving one. In reference [16] the complete one-loop corrections were calculated. These are big enough to avoid the mass bounds for scalars at the LHC.

If only the loop corrections are included, the R-parity can be conserved and the vacuum expectation value $(\mathrm{VEV})$ of the $S U(2)_{R}$ breaking triplet, $v_{R}$, remains at the $\mathrm{TeV}$ scale, leading to dark matter particle and experimentally accessible model specific particles. The scale $v_{R}$ needs to be large so that the right gauge bosons avoid experimental bounds. On the other hand, a large $v_{R}$ lifts the value of the scalar potential and large values of $v_{R}$ may lift it above the charge breaking potential. The role of the singlet in the symmetry breaking is to shift the left-right symmetry breaking scale to larger values [17]. This may happen, if the terms proportional to $v_{R}$ and to the singlet VEV $v_{S}$ in the scalar potential happen to cancel suitably, see reference [18].

If nonrenormalizable terms are not included, the $S U(2)_{R}$ gauge bosons mass scale remains accessible for future colliders. In reference [19] $W_{R}$ seemed lighter than around $1 \mathrm{TeV}$, which can be relaxed if a singlet is added [17]. Also with R-parity breaking, the model favours $W_{R}$ mass lighter than $\mathcal{O}$ (a few $10 \mathrm{TeV}$ ) [20]. An LRSUSY model with a $\mathrm{TeV}$-scale $S U(2)_{R}$ breaking scale has always a reasonably light doubly charged scalar. This was studied in the case when vacuum conserves electric charge at the tree-level with $\left\langle\tilde{\nu}_{R}\right\rangle \neq 0$ in reference [21], with non-renormalizable terms in reference [22], and finally in the case where electric charge conservation of the vacuum emerges because of loop corrections in reference [15]. In the first and last case it turns out that the right-handed scale must be around $\mathrm{TeV}$-scale, while with the non-renormalizable terms the scale is above $\mathcal{O}\left(10^{11} \mathrm{GeV}\right)$.

The discovery of a Higgs boson [23,24] gave a new handle for studies on Beyond the Standard Model (BSM) physics. The very interesting mass of the discovered scalar, around $125 \mathrm{GeV}$, is somewhat light by the Standard Model expectations, and close to the upper limit of the MSSM expectations. The extensions of MSSM usually accommodate the mass easier, in LRSUSY e.g. $[25,26]$. One of the most promising places to search for new physics is the Higgs decay branching ratios. Since the LRSUSY models with $B-L= \pm 2$ triplets always contain doubly charged Higgs bosons, $H^{ \pm \pm}$, one could expect their contribution to the $\gamma \gamma$ width to be large. However, in LRSUSY 
models, where the neutrino mass generation is dominantly of seesaw type-I, it turns out that the singly charged Higgs contribution dominates over $H^{ \pm \pm}$[27]. In the first measurements at the LHC the $\gamma \gamma$ mode seemed to differ from the SM measurement $[28,29]$, but since then the rates have converged to the SM expected rates within the experimental accuracy [30].

We will discuss in this review the minimal model with triplets breaking the $S U(2)_{R}$ symmetry and loop-corrections taking care of electric charge conservation in the vacuum. The promising particles for searches special for the minimal LRSUSY model include doubly charged Higgses $H^{ \pm \pm}$, as discussed earlier. Also their supersymmetric partners, the doubly charged higgsinos, can be accessible for experiments and signal the existence of an extended model. Their mass is given by a soft mass parameter, and can thus also be light [12-14,22]. The doubly charged higgsinos at colliders have been studied in references [31-35]. Observation of a gauge boson of the $S U(2)_{R}, W_{R}^{ \pm}$or $Z_{R}$, and the right-handed neutrinos would strongly hint of the existence of a left-right symmetric model. Some early phenomenological studies include [36-38].

In addition to the neutrinos, a problem with the Standard Model is that it does not contain a particle suitable for Dark Matter, which has been observed in the Universe $[39,40]$. The neutralino dark matter, either gaugino-dominated or higgsinodominated, has been studied in MSSM and in several extensions. In LRSUSY the neutralino dark matter was studied in reference [41] for parameter regions which allow pure bino, right-wino or triplet higgsino dark matter. The LRSUSY model contains twelve neutralinos, and in addition to gauginos and a triplet higgsino, also a doublet higgsino can be a viable and natural dark matter candidate [42], as we will discuss. In LRSUSY models the partners of the right-handed neutrinos, sneutrinos, can provide the measured relic density as well [43]. Since the cascades of the supersymmetric particle decays end up to the LSP, the composition of the LSP is relevant for the experimental signal.

In this review, the minimal LRSUSY model is shortly described. Then the phenomenologically most important particles are discussed, and the constraints on the parameter space. The possible dark matter candidates are considered, and finally examples of experimental signals.

\section{A minimal left-right supersymmetric model}

The left-right supersymmetric model is based on the gauge symmetry $S U(3)_{c} \times$ $S U(2)_{L} \times S U(2)_{R} \times U(1)_{B-L}$. Here $B, L$ stand for baryon number and lepton number, respectively. As mentioned in Section 1, the R-parity, defined as $R_{P}=(-1)^{3(B-L)+2 s}$ (with $s$ being the spin of the particle) will remain conserved, if the Higgs boson, which break the gauge symmetry contain only scalars with even $B-L$ quantum number.

Since left- and right-handed fermions in the LRSUSY model are treated similarly, necessarily both left- and right-handed quarks and leptons are in doublets, and righthanded neutrino superfields exist alongside the left-handed ones:

$$
\begin{array}{rlrl}
\left(Q_{L}\right)^{i} & =\left(\begin{array}{c}
u_{L}^{i} \\
d_{L}^{i}
\end{array}\right)=\left(\mathbf{3}, \mathbf{2}, \mathbf{1}, \frac{1}{3}\right), & \left(Q_{R}\right)^{i}=\left(\begin{array}{c}
d_{R}^{i} \\
-u_{R}^{i}
\end{array}\right)=\left(\overline{\mathbf{3}}, \mathbf{1}, \mathbf{2}^{*},-\frac{1}{3}\right), \\
\left(L_{L}\right)^{i}=\left(\begin{array}{c}
\nu_{L}^{i} \\
\ell_{L}^{i}
\end{array}\right)=(\mathbf{1}, \mathbf{2}, \mathbf{1},-1), & \left(L_{R}\right)^{i}=\left(\begin{array}{c}
\ell_{R}^{i} \\
-\nu_{R}^{i}
\end{array}\right)=\left(\mathbf{1}, \mathbf{1}, \mathbf{2}^{*}, 1\right),
\end{array}
$$

where the respective representations under the $S U(3)_{c} \times S U(2)_{L} \times S U(2)_{R} \times$ $U(1)_{B-L}$ gauge symmetry have been indicated. 
In addition to the triplets, which break the $S U(2)_{R}$ symmetry, doublets are needed to form the usual Yukawa terms for charged fermions. Because both left- and righthanded fermions are in doublets, to form the Yukawa-terms bidoublets are needed. Two of the triplets are necessary to avoid anomalies, and two bidoublets are necessary to achieve the correct CKM matrix. Furthermore, because of the left-right symmetry both left and right triplets are included. For symmetry breaking, only the righttriplets are necessary. It is possible to let the left triplets to decouple from the theory with high scale for parity breaking. Including extra singlet makes it easier to fullfil experimental constraints. The Higgs superfields of the LRSUSY model are given by

$$
\begin{aligned}
& \Phi_{1}=\left(\begin{array}{cc}
\phi_{1}^{+} & \phi_{1}^{0 \prime} \\
\phi_{1}^{0} & \phi_{1}^{-}
\end{array}\right)=\left(\mathbf{1}, \mathbf{2}, \mathbf{2}^{*}, 0\right), \\
& \Phi_{2}=\left(\begin{array}{cc}
\varphi_{2}^{+} & \varphi_{2}^{0} \\
\varphi_{2}^{0 \prime} & \varphi_{2}^{-}
\end{array}\right)=\left(\mathbf{1}, \mathbf{2}, \mathbf{2}^{*}, 0\right), \\
& \Delta_{1 L}=\left(\begin{array}{cc}
\frac{\delta_{1 L}^{-}}{\sqrt{2}} & \delta_{1 L}^{0} \\
\delta_{1 L}^{--} & -\frac{\delta_{1 L}^{-}}{\sqrt{2}}
\end{array}\right)=(\mathbf{1}, \mathbf{3}, \mathbf{1},-2), \\
& \Delta_{2 L}=\left(\begin{array}{cc}
\frac{\delta_{2 L}^{+}}{\sqrt{2}} & \delta_{2 L}^{++} \\
\delta_{2 L}^{0} & -\frac{\delta_{2 L}^{+}}{\sqrt{2}}
\end{array}\right)=(\mathbf{1}, \mathbf{3}, \mathbf{1}, 2), \\
& \Delta_{1 R}=\left(\begin{array}{cc}
\frac{\delta_{1 R}^{-}}{\sqrt{2}} & \delta_{1 R}^{0} \\
\delta_{1 R}^{--} & -\frac{\delta_{1 R}^{-}}{\sqrt{2}}
\end{array}\right)=(\mathbf{1}, \mathbf{1}, \mathbf{3},-2) \\
& \Delta_{2 R}=\left(\begin{array}{cc}
\frac{\delta_{2 R}^{+}}{\sqrt{2}} & \delta_{2 R}^{++} \\
\delta_{2 R}^{0} & -\frac{\delta_{2 R}^{+}}{\sqrt{2}}
\end{array}\right)=(\mathbf{1}, \mathbf{1}, \mathbf{3}, 2), \\
& S=(\mathbf{1}, \mathbf{1}, \mathbf{1}, 0)
\end{aligned}
$$

The corresponding superpotential is given by [43]

$$
\begin{aligned}
W= & \left(Q_{L}\right)^{T} Y_{Q}^{1} \Phi_{1}\left(Q_{R}\right)+\left(Q_{L}\right)^{T} Y_{Q}^{2} \Phi_{2}\left(Q_{R}\right)+\left(L_{L}\right)^{T} Y_{L}^{1} \Phi_{1}\left(L_{R}\right)+\left(L_{L}\right)^{T} Y_{L}^{2} \Phi_{2}\left(L_{R}\right) \\
& +\left(L_{L}\right)^{T} Y_{L}^{3} \Delta_{2 L}\left(L_{L}\right)+\left(L_{R}\right)^{T} Y_{L}^{4} \Delta_{1 R}\left(L_{R}\right)+S\left[\lambda_{L} \operatorname{Tr}\left(\Delta_{1 L} \cdot \Delta_{2 L}\right)\right. \\
& +\lambda_{R} \operatorname{Tr}\left(\Delta_{1 R} \cdot \Delta_{2 R}\right)+\lambda_{3} \operatorname{Tr}\left(\Phi_{1}^{T} \tau_{2} \Phi_{2} \tau_{2}\right)+\lambda_{4} \operatorname{Tr}\left(\Phi_{1}^{T} \tau_{2} \Phi_{1} \tau_{2}\right) \\
& \left.+\lambda_{5} \operatorname{Tr}\left(\Phi_{2}^{T} \tau_{2} \Phi_{2} \tau_{2}\right)+\lambda_{S} S^{2}+\xi_{F}\right]
\end{aligned}
$$

where generation indices are suppressed for clarity. Following the conventions of reference [44], the Yukawa couplings $Y_{Q}^{j}$ and $Y_{L}^{j}$ are $3 \times 3$ matrices in flavour space, the $\lambda$ parameters denote various trilinear Higgs interactions $\left(\tau_{2}\right.$ is the second Pauli matrix) and $\xi$ a linear singlet term. The bilinear supersymmetric Higgs mass terms are omitted in (3), but they will be generated if the singlet $S$ generates a vacuum expectation value (VEV).

The gauge symmetry is spontaneously broken when the neutral components of the Higgs multiplets generate $\mathrm{VEVs}$,

$$
\begin{aligned}
& \langle S\rangle=\frac{v_{S}}{\sqrt{2}} e^{i \alpha_{S}}, \quad\left\langle\Phi_{1}\right\rangle=\left(\begin{array}{c}
0 \frac{v_{1}^{\prime}}{\sqrt{2}} e^{i \alpha_{1}} \\
\frac{v_{1}}{\sqrt{2}} \\
0
\end{array}\right), \quad\left\langle\Phi_{2}\right\rangle=\left(\begin{array}{cc}
0 & \frac{v_{2}}{\sqrt{2}} \\
\frac{v_{2}^{\prime}}{\sqrt{2}} e^{i \alpha_{2}} & 0
\end{array}\right) \\
& \left\langle\Delta_{1 R}\right\rangle=\left(\begin{array}{cc}
0 & \frac{v_{1 R}}{\sqrt{2}} \\
0 & 0
\end{array}\right), \quad\left\langle\Delta_{2 R}\right\rangle=\left(\begin{array}{cc}
0 & 0 \\
\frac{v_{2 R}}{\sqrt{2}} & 0
\end{array}\right)
\end{aligned}
$$

the LH triplets $\Delta_{1 L}, \Delta_{2 L}$ are assumed to be inert, which is favoured by the constraints arising from the $\rho$ parameter. 
The soft SUSY breaking Lagrangian is given as [43]

$$
\begin{aligned}
\mathcal{L}_{\text {soft }}= & -\frac{1}{2}\left[M_{1} \tilde{B} \tilde{B}+M_{2 L} \tilde{W}_{L}^{a} \tilde{W}_{L a}+M_{2 R} \tilde{W}_{R}^{a} \tilde{W}_{R a}+M_{3} \tilde{g}^{a} \tilde{g}_{a}+\text { h.c. }\right] \\
& -m_{\Delta 1 L}^{2} \operatorname{Tr}\left(\Delta_{1 L}^{\dagger} \Delta_{1 L}\right)-m_{\Delta 2 L}^{2} \operatorname{Tr}\left(\Delta_{2 L}^{\dagger} \Delta_{2 L}\right)-m_{\Delta 1 R}^{2} \operatorname{Tr}\left(\Delta_{1 R}^{\dagger} \Delta_{1 R}\right) \\
& -m_{\Delta 2 R}^{2} \operatorname{Tr}\left(\Delta_{2 R}^{\dagger} \Delta_{2 R}\right)-m_{\Phi 1}^{2} \operatorname{Tr}\left(\Phi_{1}^{\dagger} \Phi_{1}\right)-m_{\Phi 2}^{2} \operatorname{Tr}\left(\Phi_{2}^{\dagger} \Phi_{2}\right)-m_{S}^{2}|S|^{2} \\
& +m_{\tilde{Q}_{L}}^{2} \tilde{Q}_{L}^{\dagger} \tilde{Q}_{L}-m_{\tilde{Q}_{R}}^{2} \tilde{Q}_{R}^{\dagger} \tilde{Q}_{R}-m_{\tilde{L}_{L}}^{2}\left(\tilde{L}_{L}^{\dagger} \tilde{L}_{L}\right)-m_{\tilde{L}_{R}}^{2}\left(\tilde{L}_{R}^{\dagger} \tilde{L}_{R}\right) \\
& -\left\{S \left[T_{L} \operatorname{Tr}\left(\Delta_{1 L} \Delta_{2 L}\right)+T_{R} \operatorname{Tr}\left(\Delta_{1 R} \Delta_{2 R}\right)+T_{3} \operatorname{Tr}\left(\Phi_{1}^{T} \tau_{2} \Phi_{2} \tau_{2}\right)\right.\right. \\
& \left.\left.+T_{4} \operatorname{Tr}\left(\Phi_{1}^{T} \tau_{2} \Phi_{1} \tau_{2}\right)+T_{5} \operatorname{Tr}\left(\Phi_{2}^{T} \tau_{2} \Phi_{2} \tau_{2}\right)+T_{S} S^{2}+\xi_{S}\right]+ \text { h.c. }\right\} \\
& +\left\{T_{Q}^{1}\left(\tilde{Q}_{L}\right)^{T} \Phi_{1}\left(\tilde{Q}_{R}\right)+T_{Q}^{2}\left(\tilde{Q}_{L}\right)^{T} \Phi_{2}\left(\tilde{Q}_{R}\right)+T_{L}^{1}\left(\tilde{L}_{L}\right)^{T} \Phi_{1}\left(\tilde{L}_{R}\right)\right. \\
& \left.+T_{L}^{2}\left(\tilde{L}_{L}\right)^{T} \Phi_{2}\left(\tilde{L}_{R}\right)+T_{L}^{3}\left(\tilde{L}_{L}\right)^{T} \Delta_{2 L}\left(\tilde{L}_{L}\right)+T_{L}^{4}\left(\tilde{L}_{R}\right)^{T} \Delta_{1 R}\left(\tilde{L}_{R}\right)+\text { h.c. }\right\},
\end{aligned}
$$

and includes gaugino mass terms (first bracket), scalar mass terms (the $m^{2}$ terms) and trilinear scalar interactions whose strengths are given by the $T$ couplings. For consistency with the superpotential, a linear $\xi$ term has also been introduced.

The $v_{i R}, v_{1}, v_{2}, v_{1}^{\prime}, v_{2}^{\prime}$ and $v_{S} \mathrm{VEVs}$ can be chosen real and non-negative, while the only complex phases which cannot be rotated away by means of suitable gauge transformations and field redefinitions are denoted by the explicit angles $\alpha_{1}, \alpha_{2}$ and $\alpha_{s}$. However, the $C P$-violating $W_{L}^{ \pm}-W_{R}^{ \pm}$mixing is proportional to $v_{1} v_{1}^{\prime} e^{i \alpha_{1}}$ and $v_{2} v_{2}^{\prime} e^{i \alpha_{2}}$, and is constrained to be small by $K^{0}-\bar{K}^{0}$ mixing data. In the following, it is assumed that

$$
v_{S}, v_{1 R}, v_{2 R} \gg v_{2}, v_{1}, v_{1}^{\prime}, v_{2}^{\prime} \quad \text { and } \quad v_{1}^{\prime}=v_{2}^{\prime}=\alpha_{1}=\alpha_{2}=\alpha_{S} \approx 0 \text {. }
$$

This choice originates from the existing constraints on the $S U(2)_{R}$ gauge bosons that impose the right sector VEVs to be large. In the supersymmetric limit, the $F$-terms and $D$-terms vanish, when $\lambda_{R} v_{1 R} v_{2 R}=\xi_{F}$ and $v_{1 R}=v_{2 R}$ [15]. On the other hand, the singlet VEV $v_{S}$ is induced by the SUSY-breaking linear term $\xi_{S}$ so that its natural scale is the supersymmetry-breaking scale. The ad-hoc hierarchy $v_{1}, v_{2} \gg v_{1}^{\prime}, v_{2}^{\prime} \approx 0$ is realized by setting $\lambda_{4}$ and $\lambda_{5}$ and the corresponding SUSY-breaking parameters small. This is a convenient setup where one, for instance, avoids potentially large flavour-changing neutral currents.

The relevant $D$-terms in the scalar potential are

$$
\begin{aligned}
V_{D}= & \sum_{i}\left[\frac{g_{L}^{2}}{8}\left|\operatorname{Tr}\left(2 \Delta_{1 L}^{\dagger} \tau_{i} \Delta_{1 L}+2 \Delta_{2 L}^{\dagger} \tau_{i} \Delta_{2 L}+\Phi_{a} \tau_{i}^{T} \Phi_{b}^{\dagger}\right)+\tilde{L}_{L}^{\dagger} \tau_{i} \tilde{L}_{L}\right|^{2}\right. \\
& \left.+\frac{g_{R}^{2}}{8}\left|\operatorname{Tr}\left(2 \Delta_{1 R}^{\dagger} \tau_{i} \Delta_{1 R}+2 \Delta_{2 R}^{\dagger} \tau_{i} \Delta_{2 R}+\Phi_{a}^{\dagger} \tau_{i}^{T} \Phi_{b}\right)+\tilde{L}_{R}^{\dagger} \tau_{i} \tilde{L}_{R}\right|^{2}\right] \\
& +\frac{g_{B-L}^{2}}{2}\left[\operatorname{Tr}\left(-\Delta_{1 L}^{\dagger} \Delta_{1 L}+\Delta_{2 L}^{\dagger} \Delta_{2 L}-\Delta_{1 R}^{\dagger} \Delta_{1 R}+\Delta_{2 R}^{\dagger} \Delta_{2 R}\right)-\tilde{L}_{L}^{\dagger} \tilde{L}_{L}+\tilde{L}_{R}^{\dagger} \tilde{L}_{R}\right]^{2}
\end{aligned}
$$

from which the coupling between the SM-like Higgs boson and the imaginary parts of right sneutrino fields can be found. Neglecting the small neutrino Yukawa couplings 
one finds

$$
\lambda_{h \tilde{\nu}_{R I} \tilde{\nu}_{R I}}=\frac{1}{4} g_{R}^{2} v \sin (\alpha+\beta) \simeq-\frac{1}{4} g_{R}^{2} v \cos 2 \beta
$$

where $\alpha$ stands for the mixing angle between the $\phi_{1}^{0}$ and $\varphi_{2}^{0}$ fields, $\tan \beta=v_{2} / v_{1}$ and where the approximated form holds in the alignment limit. This coupling is essential when computing the dark matter annihilation rates in the case of a right sneutrino LSP. It is assumed that $g_{R} \approx g_{L}$. Then at moderate or large values of $\tan \beta$, i.e. $\cos 2 \beta \simeq-1$, the coupling $\lambda_{h \tilde{\nu}_{R I} \tilde{\nu}_{R I}}$ is practically a known number.

The masses and mixings of the Higgs sector were extensively analyzed in references $[27,45]$. In reference [43] and in reference [42] the charged slepton, sneutrino, neutralino and chargino sectors were considered, as well as the collider signals with multilepton final states. The reference [18] will report on the possibility to confirm the model at a hadron collider with $\sqrt{s} \geq 27 \mathrm{TeV}$. For calculation of the particle spectrum well known tools SPHENO-3.3.8 [46] and SARAH [47] have been used, with the method introduced in reference [16] for calculating the doubly charged Higgs mass reliably.

\subsection{Higgs sector}

The Higgs boson spectrum of the minimal LRSUSY model discussed here was extensively analyzed in reference [45], including flavour constraints from $K^{0}-\bar{K}^{0}, D^{0}-\bar{D}^{0}$ and $B_{d, s}^{0}-\bar{B}_{d, s}^{0}$. The Higgs sector is rather predictable after the constraints due to the flavour changing neutral currents (FCNC) and the experimental mass bounds are taken into account. The $125 \mathrm{GeV}$ Higgs boson is the lightest particle in the Higgs sector, and the second lightest is the doubly charged Higgs boson composed of the fields in a right triplet. The lightest neutral Higgs boson is composed of the fields in one of the doublets. The large Higgs sector allows tree-level FCNC, but it was shown in reference [45] that the corresponding Higgses can be heavy, $\mathcal{O}(100 \mathrm{TeV})$, while the spectrum includes possibly TeV-scale tree-level FCNC conserving next-tolightest scalar and the lightest pseudoscalar, and the lightest charged Higgs pair, which are all mainly composed of one of the doublets, and are almost degenerate. Examples of spectra and compositions are shown in references $[27,45]$. It is also possible that the Higgs spectrum accessible to the LHC includes only the doubly charged Higgs boson in addition to the $125 \mathrm{GeV}$ Higgs boson [18].

With moderate values for $\tan \beta$ the bounds stemming from both the direct heavy Higgs boson searches in the $H / A \rightarrow \tau \tau$ channel $[48,49]$ are weaker and the contributions to the rare $B_{s} \rightarrow \mu \mu$ decay are smaller than for large $\tan \beta$. This suppresses the mixing in the neutral Higgs sector, which may challenge the SM-nature of the lightest state and lead to a large deviation from the SM for the $h \rightarrow b \bar{b}$ branching ratio [27].

The upper limit on the tree-level mass of the SM-like Higgs-boson can be much larger than in the MSSM by the virtue of the extended gauge sector. If $g_{L}=g_{R}$, one finds [50]

$$
m_{h}^{\text {tree }} \leq \sqrt{2} m_{W} \simeq 113.7 \mathrm{GeV}
$$

a value that can be easily lifted to about $125 \mathrm{GeV}$ by incorporating the radiative corrections and by adjusting the stop masses and mixing. The latter depends on $\tan \beta$, and for values close to one, the tree-level mass of the lightest scalar boson tends to vanish, like in the MSSM. However, the LRSUSY D-terms can increase the tree-level Higgs mass beyond values that are typical from the MSSM. Focusing on the 
rest of the Higgs sector, the second $C P$-even, the lightest $C P$-odd and the lightest singly-charged Higgs boson can have masses $\mathcal{O}(\mathrm{TeV})$. Close to the alignment limit, their dominant decay modes involve third generation fermions and the related LHC reach is thus similar as for the heavier states of the MSSM.

Turning to singly charged Higgs bosons, indirect constraints originating from $b \rightarrow s \gamma$ data [51] suggest that they must be heavy [52], at least if there are no cancellations in the SUSY loop-contributions to the single-charged Higgs-boson mass. This can be accommodated in LRSUSY setups if $\tan \beta_{R}=v_{2 R} / v_{1 R}$ deviates from one and if $v_{R}$ is large. Such a deviation will subsequently impact one of the diagonal elements of the doubly-charged Higgs mass matrix, making it smaller, and render the task of satisfying the doubly-charged Higgs mass constraints more difficult. We therefore adopted in reference [27]

$$
\tan \beta_{R} \simeq 1.05
$$

which, with values for $v_{R}=$ a few TeV, pushes all the MSSM-like Higgs states to be heavier than current LHC bounds. They have masses squared proportional to $g_{R}^{2} v_{R}^{2}\left(\tan ^{2} \beta_{R}-1\right)$ and are hence around the $\mathrm{TeV}$ scale. Moreover, all additional scalar bosons have masses of the order of $v_{R}, v_{S}$, or of the LH triplet soft mass parameters and hence are a lot heavier.

In the LRSUSY model building, the doubly-charged Higgs plays a central role. At the tree-level, the mass matrix has a negative eigenvalue, and thus loop-level corrections to the mass are of prime importance $[15,16]$. The most important corrections include the positive corrections from $W_{R} / H^{ \pm}$and doubly charged higgsino/neutralino, which raise the mass to experimentally allowed values. The singlino part in the neutralino is important for producing the positive contribution. With very large $v_{R}$, the negatively contributing loops, e.g. the loops proportional to the Yukawa coupling of the right triplet term, $Y_{L}^{4}$, render the mass of the doubly charged Higgs tachyonic [16]. The lepton-slepton loops play an important role, and at the same time, the relevant coupling in the loop calculation determines the decay pattern to leptons. The balance must be found to satisfy the LHC bounds. The LHC bounds for the decay of the doubly charged Higgs into electrons and muons are strong, while the di-tau final state is less constrained [53-55], due to the efficiency of tau identification. Compared to the production of the left triplets, the production of the right triplets is suppressed, since they cannot be produced together with $W_{L}^{ \pm}$and in the pair production the triplet Higgs couples to the $Z$-boson only through the $B-L$ and $W_{3 R}$ components.

One may assume that the triplet Higgs superfield mostly couples to the third generation (s)leptons, non-zero couplings to the other generations are needed to generate masses for the right-handed neutrinos. Fixing the branching ratio of the doubly-charged Higgs to electrons and muons to less than $10 \%$ helps to satisfy both the doubly charged Higgs mass constraint and to generate masses for right-handed neutrinos. The one-loop corrections to the doubly charged Higgs mass from leptonslepton loop turn negative if slepton masses are much smaller than $v_{R}[45,56]$, and thus heavy right sleptons are favoured.

\subsection{Gauge sector}

In the minimal LRSUSY model the charged $S U(2)_{R}$ gauge boson $W_{R}$ is always lighter than the neutral $Z_{R}$, and thus $W_{R}$ is the more constraining of the two. The parameters in the model are intertwined in such a way that $v_{R} \leq 15 \mathrm{TeV}[16,18]$ because of the need to stabilize the charge conserving vacuum by loop corrections. This bound would be smaller, were there not the assumed singlet [17]. The experimental bounds from 
from dijet searches at LHC are $m_{W_{R}}>3.6 \mathrm{TeV}$ (ATLAS) [57] and $>3.3 \mathrm{TeV}$ (CMS) [58]. These can be used in LRSUSY, if $g_{L}=g_{R}$ and no new decay channels are open. However, the gauge bosons can easily possess additional decay modes to a pair of lighter supersymmetric particles (usually electroweakinos or sleptons) or to some of the new scalar bosons. All these new modes invariably affect the total decay width and the branching ratios of these gauge bosons so that the existing limits cannot be directly/blindly applied. The discussion on relaxation of the bounds is in reference [16].

The $W_{R}$-boson decays into right-handed leptons and neutrinos, if this decay channel is open. Via resonant production of $W_{R}$, the production of right-handed neutrinos can be substantial. The Majorana nature of the right-handed neutrino $N_{R}$ allows same rate for the decay products to positively and negatively charged leptons. From the process [59]

$$
p p \rightarrow W_{R} \rightarrow N_{R}\left(\rightarrow \ell W_{R}^{*}\right) \ell \rightarrow \ell \ell j j,
$$

CMS has derived contours with $\ell=e$ and $\ell=\mu$ for the plane $\left(M_{W_{R}}, M_{N_{R}}\right)$ [60]. The bounds are stronger for the neutrino masses in the $[400 \mathrm{GeV}, 1 \mathrm{TeV}]$ window, as the $W_{R}$-boson is constrained to be heavier than $3.3 \mathrm{TeV}$. In contrast, for setups with neutrinos lighter than $200 \mathrm{GeV}$, the $W_{R}$-bosons are constrained only by resonance searches in the dijet mode. Of course, also the lower bounds extracted from LEP data must be fulfilled, with the right-handed Majorana neutrino heavier than about $90 \mathrm{GeV}[61]$.

The standard search above assumes the $N_{R}$ to decay $100 \%$ via $W_{R}$ [60]. In LRSUSY also the decay $N_{R} \rightarrow \ell^{ \pm} H^{\mp}$ needs to be considered, as well as supersymmetric decay modes. If kinematically allowed, the favoured decay mode is $N_{R} \rightarrow \ell H$

followed by $H \rightarrow t \bar{b}$. These follow from small triplet component in $H^{ \pm}$, and can be dominant, if $W_{R}$ is strongly off-shell. This way the reported exclusion limits from CMS [60] become less severe, as shown in reference [16].

Eventually, the LHC will probe higher $W_{R}$ masses. It has been shown that the discovery of $W_{R}$ bosons with masses reaching up to $5 \mathrm{TeV}$ and their exclusion for masses as large as $6 \mathrm{TeV}$ could be achieved with $300 \mathrm{fb}^{-1}$ of proton-proton collisions at a centre-of-mass energy of $\sqrt{s}=14 \mathrm{TeV}$ [62-64]. Our LRSUSY parameterization allows for stable vacua featuring a heavy $W_{R}$ boson with a mass ranging up to about $8 \mathrm{TeV}$. The total exclusion of $W_{R}$ bosons predicted in LRSUSY models nevertheless requires a higher collision energy than the one available at the LHC, as there will always remain parts of the parameter space where the $W_{R}$ boson can escape detection, see reference [43].

\section{Lightest supersymmetric particles}

Neutralinos and right-sneutrinos are among the key particles in the model, as either can be the lightest supersymmetric particle (LSP) in the model. Because of the automatic R-parity conservation, the LSP is a dark matter candidate, as it always provides at least part of the relic density of the universe. In addition, in the decay of any other supersymmetric particle LSP is the remaining stable particle in addition to the Standard Model particles.

\subsection{The neutralino and chargino sector}

Detailed information of the chargino and neutralino sector of the model is reported in reference [44]. 
The model has six singly-charged charginos whose associated mass matrix can be written in the $\left(\tilde{\Delta}_{L}^{ \pm}, \tilde{\Delta}_{R}^{ \pm}, \tilde{\Phi}_{1}^{ \pm}, \tilde{\Phi}_{2}^{ \pm}, \tilde{W}_{L}^{ \pm}, \tilde{W}_{R}^{ \pm}\right)$basis as

$$
M_{\tilde{\chi}^{ \pm}}=\left(\begin{array}{cccccc}
\lambda_{L} v_{s} / \sqrt{2} & 0 & 0 & 0 & 0 & 0 \\
0 & \lambda_{R} v_{s} / \sqrt{2} & 0 & 0 & 0 & -g_{R} v_{1 R} \\
0 & 0 & 0 & \mu_{\mathrm{eff}} & g_{L} v_{u} / \sqrt{2} & 0 \\
0 & 0 & \mu_{\mathrm{eff}} & 0 & 0 & -g_{R} v_{d} / \sqrt{2} \\
0 & 0 & 0 & g_{L} v_{d} / \sqrt{2} & M_{2 L} & 0 \\
0 & g_{R} v_{2 R} & -g_{R} v_{u} / \sqrt{2} & 0 & 0 & M_{2 R}
\end{array}\right),
$$

where $v_{u}=v \sin \beta, v_{d}=v \cos \beta$.

If the LSP is from the neutralino sector, it can be either a gaugino or a higgsino. The model contains twelve neutralinos, and the neuralino mass matrix can be arranged into three block diagonal pieces, when left triplet is inert, as well as two neutral bidoublet Higgs bosons. The first two blocks are expressed, in the $\left(\tilde{\delta}_{1 L}, \tilde{\delta}_{2 L}\right)$ and $\left(\tilde{\phi}_{2}, \tilde{\varphi}_{1}\right)$ bases, as

$$
M_{\tilde{\chi} \delta}=\left(\begin{array}{cc}
0 & \mu_{L} \\
\mu_{L} & 0
\end{array}\right) \quad \text { and } \quad M_{\tilde{\chi}_{\Phi}}=\left(\begin{array}{cc}
0 & -\mu_{\mathrm{eff}} \\
-\mu_{\mathrm{eff}} & 0
\end{array}\right),
$$

while the last block reads, in the $\left(\tilde{\phi}_{1}, \tilde{\varphi}_{2}, \tilde{\delta}_{1 R}, \tilde{\delta}_{2 R}, \tilde{S}, \tilde{B}, \tilde{W}_{L}^{0}, \tilde{W}_{R}^{0}\right)$ basis,

$$
M_{\tilde{\chi}^{0}}=\left(\begin{array}{cccccccc}
0 & -\mu_{\mathrm{eff}} & 0 & 0 & -\mu_{d} & 0 & \frac{g_{L} v_{u}}{\sqrt{2}} & -\frac{g_{R} v_{u}}{\sqrt{2}} \\
-\mu_{\mathrm{eff}} & 0 & 0 & 0 & -\mu_{u} & 0 & -\frac{g_{L} v_{d}}{\sqrt{2}} & \frac{g_{R} v_{d}}{\sqrt{2}} \\
0 & 0 & 0 & \mu_{R} & \frac{\lambda_{R} v_{2 R}}{\sqrt{2}} & g^{\prime} v_{1 R} & 0 & -g_{R} v_{1 R} \\
0 & 0 & \mu_{R} & 0 & \frac{\lambda_{R} v_{1 R}}{\sqrt{2}} & -g^{\prime} v_{2 R} & 0 & -g_{R} v_{2 R} \\
-\mu_{d} & -\mu_{u} & \frac{\lambda_{R} v_{2 R}}{\sqrt{2}} & \frac{\lambda_{R} v_{1 R}}{\sqrt{2}} & \mu_{S} & 0 & 0 & 0 \\
0 & 0 & g^{\prime} v_{R} & -g^{\prime} v_{2 R} & 0 & M_{1} & 0 & 0 \\
\frac{g_{L} v_{u}}{\sqrt{2}} & -\frac{g_{L} v_{d}}{\sqrt{2}} & 0 & 0 & 0 & 0 & M_{2 L} & 0 \\
-\frac{g_{R} v_{u}}{\sqrt{2}} & \frac{g_{R} v_{d}}{\sqrt{2}} & -g_{R} v_{1 R} & -g_{R} v_{2 R} & 0 & 0 & 0 & M_{2 R}
\end{array}\right),
$$

where we have defined $\mu_{S}=\lambda_{S} \frac{v_{s}}{\sqrt{2}}, \mu_{L, R}=\lambda_{L, R} \frac{v_{s}}{\sqrt{2}}$ and $\mu_{u, d}=\lambda_{3} \frac{v_{u, d}}{\sqrt{2}}$.

The relations between gaugino soft masses, $\mu_{s}$ and $\mu_{\text {eff }}$ determine which of the neutralinos is the lightest. An interesting situation arises, when $\mu_{\text {eff }}$ value leads to LSP, which is a doublet higgsino, since there will then always be four almost mass degenerate neutralinos and two charginos.

\subsection{The lepton-slepton sector}

If sneutrino is the LSP, the gauginos and higgsinos can be made heavy by having large gaugino soft masses. The singlet self-coupling $\lambda_{s}$ needs to be large enough to prevent singlino LSP, and the VEVs $v_{1 R}, v_{2 R}$ and $v_{s}$ relatively large. by

In the basis $\left(\tilde{L}_{L}^{i}, \tilde{L}_{R}^{i}\right)$, the squared-mass matrix for the charged sleptons is given

$$
\mathcal{M}_{L}^{2}=\left(\begin{array}{cc}
m_{\tilde{L}_{L}}^{2}+m_{\ell}^{2}+D_{11} & \left(T_{L}^{3}\right)_{i j} v \cos \beta+\mu_{\text {eff }} m_{\ell} \tan \beta \\
\left(T_{L}^{3}\right)_{i j} v \cos \beta+\mu_{\text {eff }} m_{\ell} \tan \beta & m_{\tilde{L}_{R}}^{2}+m_{\ell}^{2}+D_{22}
\end{array}\right)
$$


where $\mu_{\text {eff }}=\lambda_{3} v_{s} / \sqrt{2}$ and the $D$-terms read

$$
\begin{aligned}
& D_{11}=-\frac{g_{L}^{2}}{8} v^{2} \cos 2 \beta+g_{B-L}^{2}\left(v_{1 R}^{2}-v_{2 R}^{2}\right) \text { and } \\
& D_{22}=\frac{g_{R}^{2}}{8}\left[2\left(v_{1 R}^{2}-v_{2 R}^{2}\right)-v^{2} \cos 2 \beta\right]-g_{B-L}^{2}\left(v_{1 R}^{2}-v_{2 R}^{2}\right) .
\end{aligned}
$$

The scalar and pseudoscalar sneutrino mixing matrices are of the form

$$
\mathcal{M}_{\tilde{\nu}}^{2}=\left(\begin{array}{ll}
M_{\tilde{\nu}_{L} \tilde{\nu}_{L}}^{2} & M_{\tilde{\nu}_{L} \tilde{\nu}_{R}}^{2} \\
M_{\tilde{\nu}_{R} \tilde{\nu}_{L}}^{2} & M_{\tilde{\nu}_{R} \tilde{\nu}_{R}}^{2}
\end{array}\right) .
$$

The scalar mass matrix entries are

$$
\begin{aligned}
& M_{\tilde{\nu}_{L} \tilde{\nu}_{L}}^{2}=m_{\tilde{L}_{L}}^{2}+D_{11}, \\
& M_{\tilde{\nu}_{L} \tilde{\nu}_{R}}^{2}=M_{\tilde{\nu}_{R} \tilde{\nu}_{L}}^{2}=\left(T_{L}^{2} v-Y_{L}^{2} Y_{L}^{4} v_{1 R}\right) \sin \beta+Y_{L}^{2} \mu_{\mathrm{eff}} \frac{v \cos \beta}{\sqrt{2}}, \\
& M_{\tilde{\nu}_{R} \tilde{\nu}_{R}}^{2}=m_{\tilde{L}_{R}}^{2}+D_{22}+2\left(Y_{L}^{4}\right)^{2} v_{1 R}^{2}-\sqrt{2} T_{L}^{4} v_{1 R}+Y_{L}^{4} \lambda_{R} v_{S} v_{2 R},
\end{aligned}
$$

where $D_{11}$ and $D_{22}$ are given in equation (16). The terms depending on the Yukawa couplings $Y_{L}^{2}$ will be small to get viable neutrino masses. right- and left-handed neutrino mixing term will be small as well, unless $T_{L}^{2}$ is large. The pseudoscalar mass matrix elements are

$$
\begin{aligned}
& M_{\tilde{\nu}_{I L} \tilde{\nu}_{I R}}^{2}=M_{\tilde{\nu}_{I R} \tilde{\nu}_{I L}}^{2}=\left(T_{L}^{2} v+Y_{L}^{2} Y_{L}^{4} v_{1 R}\right) \sin \beta+Y_{L}^{2} \mu_{\mathrm{eff}} \frac{v \cos \beta}{\sqrt{2}} \\
& M_{\tilde{\nu}_{I R} \tilde{\nu}_{I R}}^{2}=m_{\tilde{L}_{R}}^{2}+D_{22}+2\left(Y_{L}^{4}\right)^{2} v_{1 R}^{2}+\sqrt{2} T_{L}^{4} v_{1 R}-Y_{L}^{4} \lambda_{R} v_{S} v_{2 R},
\end{aligned}
$$

with $M_{\tilde{\nu}_{I L} \tilde{\nu}_{I L}}^{2}=M_{\tilde{\nu}_{L} \tilde{\nu}_{L}}^{2}$.

With large values for $\lambda_{R}$ and positive parameters the last term of equation (21) becomes most important for the sneutrino masses. One of the pseudoscalar states, with a flavour aligned with the largest element in the $Y_{L}^{4}$ matrix, can then be the LSP unless the corresponding soft supersymmetry breaking mass term is significantly larger than the other terms.

Right sneutrinos have been investigated in detail, including implications for direct and indirect DM detection, signals at the LHC, and restrictions on the model parameter space, but mostly the studied sneutrino has been a gauge singlet, see the list of references e.g. in reference [43].

\section{Dark matter}

Any of the possible LSPs discussed above, a right sneurino, a gaugino or a higgsino is a dark matter candidate for some part of the parameter space, and can satisfy the relic density measurement by the Planck collaboration. The Planck collaboration derived as the $2 \sigma$ experimentally-allowed range [40]:

$$
\Omega h^{2} \in[0.1163,0.1217]
$$

which is the dark matter energy density evaluated relatively to the critical energy density of the universe and $h$ the reduced Hubble parameter. 
In addition to the direct detection cross section from XENON1T [65] and PANDA [66], also indirect limits from FermiLAT [67] were considered in reference [42]. The direct searches constrain the parameter space strongly, while all the tested points gave thermally averaged dark matter annihilation cross section well below the FermiLAT value.

\subsection{Sneutrino dark matter}

The sneutrino LSP mostly annihilates via a SM-like Higgs-boson exchange in the $s$-channel, with the coupling given in equation (8). If the right-handed neutrinos are lighter than sneutrinos, a $t$-channel neutralino exchange diagram also exists, although it is suppressed for heavy neutralinos.

In reference [42], from which this discussion is mostly, the dark matter relic density and direct detection constraints are calculated with MADDM v2.0 [68], and with MicrOmegas [69] to validate the findings. Due to the dominance of the relic density on the $s$-channel diagrams, it only depends on the right sneutrino mass. Existing bounds are found to be satisfied with sneutrinos having a mass lying in the

$$
[250 \mathrm{GeV}, 290 \mathrm{GeV}]
$$

range (provided all co-annihilation channels are negligible). For benchmark points in reference [42], the spin-independent cross sections for dark matter-nucleon scattering was found to be smaller than $2.5 \times 10^{-10} \mathrm{pb}$ agreeing with the current bounds.

As the dark matter-nucleon scattering is mediated mostly via the SM-like Higgs boson, a direct detection signal will be largely unaffected by the details of the unknown particle spectrum and thus provides a robust way of testing right sneutrino dark matter in LRSUSY models. In presence of coannihilations or large mixing between the left and right sneutrino sectors, the observed relic density may point to another mass range than in our case. Moreover, if heavier sneutrinos are allowed, the direct detection bounds are weaker and the exclusion will be more difficult.

We could alternatively rely on the XENON1T $2 \sigma$ upper limit on $\sigma_{S I}$, given the large uncertainties that contribute to the cross-section measurements. This leads to the existence of viable configurations that would merit further attention. However, the expected progress in future dark matter direct detection experiments could potentially exclude the full hypothesis of sneutrino dark matter in left-right supersymmetry. Direct detection would indeed push for heavier sneutrinos, which turn out to yield over-abundant dark matter. Including co-annihilations may, however, modify these conclusions.

If there are superpartners that are close in mass to the LSP, they are present when dark matter freezes out and co-annihilation processes need to be taken into account [70]. Charginos and neutralinos annihilate more efficiently to SM particles than sneutrinos. Co-annihilations consequently reduce the relic density relative to the no-co-annihilation case, although the effect is Boltzmann-suppressed when the mass difference between the LSP and the co-annihilating particles becomes larger. Consider co-annihilations of the sneutrino with NLSP neutralino and/or chargino states, LRSUSY models having altogether twelve neutralinos and six singly-charged charginos. While most states are naturally in the multi-TeV range, some may be lighter and thus relevant from a cosmological standpoint. Their masses are controlled by the soft supersymmetry-breaking parameters for what concerns the gauginos, while the higgsinos have a mass of the order of $\mu_{\mathrm{eff}}$.

The bidoublet higgsinos form a nearly degenerate set of four neutralinos and two charginos and hence co-annihilations are always present if the lightest of these neutralinos is either the LSP, or the NLSP in the case where it is nearly degenerate 
with the LSP. The higgsinos co-annihilate mainly via the $\tilde{\chi}_{i}^{0} \tilde{\chi}_{j}^{ \pm} \rightarrow q \bar{q}^{\prime}$ and $\tilde{\chi}_{i}^{0} \tilde{\chi}_{j}^{0} \rightarrow q \bar{q}$ or $V V(V=W, Z)$ channels, processes that are all mediated mainly by $s$-channel $W$ boson, $Z$ boson, and Higgs boson exchanges with the mediator depending on the charges and $C P$ properties of the co-annihilating particles. Annihilations into quarks via gauge boson exchanges are often the dominant channels and the relevant couplings are standard electroweak gauge couplings.

If a sneutrino LSP is mostly degenerate with the higgsinos, co-annihilations with the sneutrino need to be considered. The most significant of these modes consists in $\tilde{\nu} \tilde{\chi}^{0} \rightarrow \ell^{ \pm} W^{\mp}$ co-annihilations, which proceed via a $t$-channel wino exchange. Since this channel requires either a mixing between the left- and right- sneutrinos, or between the left- and right-handed charged winos, both mixings that are small in our model, the corresponding contributions to the relic abundance are relatively small compared to the $\tilde{\nu} \tilde{\nu} \rightarrow V V$ or $t \bar{t}$ modes. For cases in which the splittings between the sneutrino LSP and the lighter neutralinos and charginos are small, it however turns out that neutralino-pair and neutralino-chargino annihilation cross sections are one order of magnitude larger than the sneutrino-sneutrino one (provided the Boltzmann suppression is not too important).

The dependence of the mass difference between the sneutrino LSP and the NLSP on the sneutrino mass is such that cosmologically-viable configurations can be found for mostly any sneutrino mass ranging up to $675 \mathrm{GeV}$, the mass value at which the sneutrino cannot be the LSP anymore. The LSP mass can hence be viably shifted by up to several hundreds of $\mathrm{GeV}$ by the sole virtue of the co-annihilation channels.

For many scanned configurations, the co-annihilating new degrees of freedom annihilate less efficiently than the LSP. Their net effect is a reduction of the full annihilation rate at freeze-out instead of an enhancement, so that the relic density is increased [71]. In LRSUSY setups with a sneutrino LSP, this happens either when some of the heavier Higgs bosons are lighter than the LSP, or when the spectrum features nearly degenerate sneutrinos. In addition to the SM-like Higgs boson $h$, LRSUSY spectra indeed always feature MSSM-like Higgs states (namely a $C P$-even Higgs boson $H$, a $C P$-odd Higgs boson $A$ and a charged Higgs boson $H^{ \pm}$) which are nearly degenerate. These can in principle be lighter than the LSP and then impact the relic density in the sneutrino LSP case through $D$-term four-point couplings that drive the $\tilde{\nu}_{R} \tilde{\nu}_{R} \rightarrow H H, A A$ and $H^{+} H^{-}$annihilation channels. While such an option allows for very light sneutrino LSP solutions with respect to the relic abundance, one cannot get a scenario where constraints from direct searches for heavier scalars and flavor physics can simultaneously be satisfied. A light $C P$-odd state indeed sizeably contributes to $B_{s} \rightarrow \mu^{+} \mu^{-}$[72], which is excluded in the light of current data. Spectra exhibiting several light and degenerate sneutrinos are however not affected by those considerations, so that one may push the sneutrino mass down to about $200 \mathrm{GeV}$. This possibility is, however, ruled out by DM direct detection bounds from XENON1T.

The viable parameter space for other types of co-annihilating scenarios (featuring light $S U(2)_{\mathrm{R}}$ wino-like or higgsino-like electroweakinos for instance) are excluded by collider searches for extra gauge bosons or doubly-charged Higgs bosons.

\subsection{Neutralino dark matter}

LRSUSY neutralino dark matter has been already discussed in the past, but under different assumptions than in reference [42] from where the discussion here is, e.g. reference [41] considers neutralinos that are pure gauge eigenstates, so that their results must be generalized to the case where neutralinos are admixtures of electroweakinos and higgsinos, as the composition of the lightest neutralino depends on the soft gaugino masses. If $M_{1}$ is the smallest gaugino mass parameter to guarantee a 
bino-dominated LSP, one can fix its value in order to obtain a relic density prediction in agreement with data. The $M_{1}$ parameter is connected to the $U(1)_{B-L}$ gaugino (that we abusively call bino), so that it does not couple to the light gauge bosons and the bidoublet Higgs fields. The bino, however, mixes with the other gauginos, which ensures non-vanishing couplings to the $Z$-boson and the SM-like Higgs-boson.

The resulting relic density is in general too large, but a resonant contribution may increase the dark matter annihilation cross section in which case the neutralino LSP mass should be slightly below half the Higgs-boson mass $m_{h} / 2$. The kinematically allowed $h \rightarrow \tilde{\chi}_{1}^{0} \tilde{\chi}_{1}^{0}$ decay is suppressed since the LSP is bino-dominated and the bidoublets are not charged under $U(1)_{B-L}$. The benchmarks constructed in reference [43] satisfy current direct detection bounds [73].

Cosmologically viable setups could be also obtained when the LSP is a neutral higgsino. In this case, the relic density increases with the LSP mass once all annihilation channels are kinematically open. The Planck value is matched for LSP masses of around $750 \mathrm{GeV}$ when co-annihilations with sneutrinos are ignored. The impact of the latter decreases the effective annihilation cross section and then points towards a slightly lighter LSP of about $675-700 \mathrm{GeV}$. This leads to viable spectra that are fairly heavy, with all lighter states being mostly degenerate bidoublet higgsinos and sneutrinos. Such a configuration would also be roughly compatible with the AMS-02 results [74], which points to a $\mathrm{TeV}$-scale $\mathrm{DM}$ candidate.

Scenarios in which a gaugino state is nearly degenerate with the higgsinos do not yield much differences. If the LSP is higgsino-dominated, with an up to $30 \%$ gaugino admixture, the relic density constraint can be satisfied with slightly lighter LSP masses, and the annihilation channels are nearly the same as in a scenario where only higgsinos would be co-annihilating. Bino-higgsino co-annihilations, that could be crucial in the MSSM, do not work in the same way in LRSUSY models in which the bino always mixes strongly with the $S U(2)_{\mathrm{R}}$ wino, so that there is no pure binostate at all. Therefore, if we try to design a scenario in which a bino-state would be degenerate with the higgsinos, the higgsinos will also mix with this bino-wino combination. Basically we will end with two states, both admixtures of gauginos and higgsinos. The mixing lifts the degeneracy among the higgsinos, so that one state will be lighter and the other heavier than the original with degenerate higgsinos. This difference with the MSSM is thus completely expected, as the MSSM $\left(U(1)_{\mathrm{Y}}\right)$ bino is here made of an admixture of the neutral $S U(2)_{\mathrm{R}}$ gaugino and the $U(1)_{B-L}$ bino. The well-tempered MSSM scenario consists thus of a triple admixture of states.

However, if the most gaugino-dominated state is the LSP, the mass difference between the co-annihilating particles is larger, so that the net effect on the relic density is Boltzmann-suppressed. The bino-wino mixture does not annihilate as efficiently as higgsinos, the corresponding relic density turns to be larger than the Planck value, despite the co-annihilations.

\section{Collider signals}

From the point of view of collider experiments, the most interesting particles in the LRSUSY model are those, which are expected to be light enough to access experimentally. The lightest doubly charged Higgs boson belongs to that category, and even provides a possibility to exclude the model. As was discussed earlier, the nextto-lightest scalar $H_{1}^{0}$, the lightest pseudoscalar $A_{1}^{0}$ and the lightest charged Higgs $H_{1}^{ \pm}$ are composed almost exclusively of fields in one doublet. Thus from the experimental point of view, they are very similar to the same mass heavy Higgses of MSSM. One might think that the light doubly charged Higgs would result in new kind of signals to search e.g. for the singly charged Higgs, but since the doubly charged Higgs is purely from triplets, the coupling between them is tiny. 
At the LHC, the doubly charged Higgs from right triplet can be produced in pairs [75] or in the fusion of right gauge bosons $W_{R}[76,77]$. In the minimal LRSUSY model discussed, $H^{ \pm \pm}$is lighter than $W_{R}$ or singly charged Higgs or supersymmetric partners into which it could decay. The only available decay mode is to a same sign pair of leptons. If the leptons are electrons or muons, the signal is straightforward, and the LHC mass limit is $\sim 800 \mathrm{GeV}$ [78]. If the decay is to taus, the LHC mass bound is weaker, $\sim 500 \mathrm{GeV}$ [55]. Reaching TeV-range in this case would not be possible even with HL-LHC. In reference [18], the search of $H^{ \pm \pm}$decaying more than $90 \%$ to a tau pair is considered. It is noticed that using multivariate analysis, it seems possible to reach heavier than $1 \mathrm{TeV} H^{ \pm \pm}$with center-of-mass energy $\geq 27 \mathrm{TeV}$.

If the doubly charged scalar is discovered, and in addition a doubly charged higgsino and an $S U(2)_{R}$ gauge boson, this would be a strong indication of leftright supersymmetry, without any other supersymmetric particle been found. Signals for these have, therefore, been studied extensively, e.g. [22,31-35,44,79]. In references $[18,42,43]$ signals have been investigated when dark matter in the minimal SUSYLR model is assumed, and some differences depending on type of dark matter are found. A specific related aspect concerns the possibility of resonantly producing supersymmetric particles at colliders, in particular due to the presence of extra gauge and Higgs bosons. In this context, dark matter constraints and the expected resonant collider signals are explored $[18,42,43]$, paying particular attention to distinguishing features.

The LHC phenomenology connected to LRSUSY neutralino and chargino states has been recently analysed in reference [44], where it has been shown that the leptonic channels are the best probes for LRSUSY neutralinos and charginos. The production rates are in general larger than in the MSSM for not too heavy gauginos, so that this additionally offers handles to distinguish the LRSUSY case from the MSSM. For a comparative study with cases where the neutralino is the LSP, we focus on LRSUSY realizations where the lightest neutralino is bino-dominated. In this case, we fix the bino soft-mass $M_{1}$ to a value yielding a dark matter relic density as measured by the Planck satellite.

For LRSUSY scenarios with a bino-dominated LSP, charged sleptons and sneutrinos both decay into the neutralino LSP and either a lepton or a right-handed neutrino with a large branching fraction. The right-handed neutrino then decays into an $\ell j j$ system so that the full decay chain is connected with a signature that includes two leptons, two jets and missing transverse energy. In the case where sneutrinos cannot decay into a $\nu_{R} \tilde{\chi}_{1}^{0}$ final state, they instead decay invisibly to a $\nu_{L} \tilde{\chi}_{1}^{0}$ system which does not yield any multileptonic final state. Decay modes exhibiting three or more leptons are rare and the corresponding number of events amounts to about $10 \%$ of the number of dilepton events, see reference [43].

When the LSP is a sneutrino, more leptons are expected in the final states, as intermediate charginos that can be produced in the longer decay chains lead to additional leptons. In this case, the number of events featuring three leptons amounts to $20-30 \%$ of the number of expected dilepton events. In high luminosity LHC, one may hope to distinguish a sneutrino LSP scenario from the corresponding neutralino LSP scenario, as for the study of the rarer same-sign dilepton signature that seems very unlikely to yield any visible signals at the low-luminosity phase of the LHC. The collider signatures of this model differ from the cases in which the right-handed sneutrino is a singlet, as now the sneutrino can couple differently, through gauge interactions.

LRSUSY sneutrino LSP scenarios present also a very different phenomenology from the corresponding MSSM scenarios where the MSSM is extended by righthanded neutrino superfields. In this last case, the Lagrangian includes Dirac mass terms for the neutrinos and the lightest stau is often close in mass to the sneutrino. 
Due to the small associated Yukawa coupling, the lightest stau is long-lived [80], contrasting the scenarios where the stau is much heavier than the sneutrinos (see Eqs. (15), (20) and (21)). In LRSUSY, the next-to-lightest superpartner (NLSP) turns thus out to be another particle, and the presence of larger couplings of the sneutrino to the other particles guarantees that the NLSP is typically not long-lived.

The presence of relatively light $S U(2)_{R}$ gauge bosons enhances the production rate of electroweakinos at the LHC, and thus higher superpartner masses can be achieved than in MSSM. Corresponding events feature, in addition, a larger amount of missing transverse momentum and at least one very hard lepton, which helps to suppress the SM background. In the case of bidoublet higgsino dark matter, the viable neutralino dark matter candidate has a mass around $700 \mathrm{GeV}[42,43]$, and four neutralinos and two charginos are almost mass degenerate. The rather heavy spectrum makes this scenario difficult to detect. As any new gauge boson or colored superpartner is heavy enough for their production rate to be suppressed, any potential collider signal becomes hard to get. Even when considering cascades such as those originating from the production of a single $W_{R}$ boson, a large integrated luminosity is necessary to observe any signal. The main $W_{R}$ boson signature consists in a production of numerous leptons, jets and missing energy.

\section{Conclusions}

Many researchers were quite disappointed when supersymmetry was not found soon after the LHC started. These expectations were based on MSSM with additional strong assumptions. Here we have discussed another minimal model, again with many assumptions, but with different starting point from MSSM.

We have assumed high energy restoration of the parity symmetry, neutrino mass generation by seesaw mechanism and that nothing but loop corrections are included in order to have an electrically stable vacuum. These assumptions lead to a model with rather light $S U(2)_{R}$ gauge bosons and a light doubly charged Higgs, and at the same time viable dark matter relic density.

Experimental observation of $W_{R}$, doubly charged Higgs and the right-handed neutrino would be a clear sign of the left-right model with seesaw mechanism. Detection of doubly charged higgsino would tell that the model is supersymmetric. In the minimal version of the model the doubly charged Higgs is relatively light, and if it exists, it will be detected in future collider experiments.

Open access funding provided by University of Helsinki including Helsinki University Central Hospital. I thank Arindam Chatterjee, Durmus Demir, Mariana Frank, Benjamin Fuks, Dilip Ghosh, Jukka Maalampi, Subhadeep Mondal, Pran Pandita, Kai Puolamäki, Santosh Rai, Martti Raidal, Nikolai Romanenko, Ipsita Saha, Ismail Turan, and Harri Waltari for collaboration on supersymmetric left-right models over the years. Recently, among other things, deep knowledge of the model by Mariana Frank, and in addition gut feeling of the spectrum as well as ability to use computational methods to study the complicated spectrum by Benjamin Fuks, Subhadeep Mondal, Santosh Rai and Harri Waltari have made the collaborations enjoyable.

Open Access This is an open access article distributed under the terms of the Creative Commons Attribution License (https://creativecommons.org/licenses/by/4.0), which permits unrestricted use, distribution, and reproduction in any medium, provided the original work is properly cited. 
Publisher's Note The EPJ Publishers remain neutral with regard to jurisdictional claims in published maps and institutional affiliations.

\section{References}

1. R.N. Mohapatra, J.C. Pati, Phys. Rev. D 11, 2558 (1975)

2. G. Senjanovic, R.N. Mohapatra, Phys. Rev. D 12, 1502 (1975)

3. P. Minkowski, Phys. Lett. B 67, 421 (1977)

4. R.N. Mohapatra, G. Senjanovic, Phys. Rev. Lett. 44, 912 (1980)

5. T. Yanagida, Conf. Proc. C 7902131, 95 (1979)

6. M. Gell-Mann, P. Ramond, R. Slansky, Conf. Proc. C 790927, 315 (1979)

7. S.L. Glashow, NATO Sci. Ser. B 61, 687 (1980)

8. R.N. Mohapatra, A. Rasin, Phys. Rev. Lett. 76, 3490 (1996)

9. R.N. Mohapatra, A. Rasin, Phys. Rev. D 54, 5835 (1996)

10. R. Kuchimanchi, Phys. Rev. Lett. 76, 3486 (1996)

11. R. Kuchimanchi, R.N. Mohapatra, Phys. Rev. D 48, 4352 (1993)

12. C.S. Aulakh, A. Melfo, A. Rasin, G. Senjanovic, Phys. Rev. D 58, 115007 (1998)

13. C.S. Aulakh, A. Melfo, G. Senjanovic, Phys. Rev. D 57, 4174 (1998)

14. B. Dutta, R.N. Mohapatra, Phys. Rev. D 59, 015018 (1999)

15. K.S. Babu, R.N. Mohapatra, Phys. Lett. B 668, 404 (2008)

16. L. Basso, B. Fuks, M.E. Krauss, W. Porod, JHEP 1507, 147 (2015)

17. M. Cvetic, Phys. Lett. B 164, 55 (1985)

18. M. Frank, B. Fuks, K. Huitu, S. Mondal, S.K. Rai, H. Waltari, Phys. Rev. D 101, $115014(2020)$

19. M. Cvetic, J.C. Pati, Phys. Lett. B 135, 57 (1984)

20. K. Huitu, P. Pandita, K. Puolamaki, Phys. Lett. B 415, 156 (1997)

21. K. Huitu, J. Maalampi, Phys. Lett. B 344, 217 (1995)

22. Z. Chacko, R.N. Mohapatra, Phys. Rev. D 58, 015003 (1998)

23. G. Aad et al. [ATLAS Collaboration], Phys. Lett. B 716, 1 (2012)

24. S. Chatrchyan et al. [CMS Collaboration], Phys. Lett. B 716, 30 (2012)

25. K. Huitu, P. N. Pandita, K. Puolamaki, Phys. Lett. B 423, 97 (1998)

26. Y. Zhang, H. An, X.d. Ji, R.N. Mohapatra, Phys. Rev. D 78, 011302 (2008)

27. M. Frank, D.K. Ghosh, K. Huitu, S.K. Rai, I. Saha, H. Waltari, Phys. Rev. D 90, 115021 (2014)

28. G. Aad et al. [ATLAS Collaboration], Phys. Rev. Lett. 108, 111803 (2012)

29. S. Chatrchyan et al. [CMS Collaboration], Phys. Lett. B 710, 403 (2012)

30. M. Tanabashi et al. [Particle Data Group], Phys. Rev. D 98, 030001 (2018)

31. M. Raidal, P.M. Zerwas, Eur. Phys. J. C 8, 479 (1999)

32. M. Frank, Phys. Rev. D 62, 053004 (2000)

33. M. Frank, K. Huitu, S.K. Rai, Phys. Rev. D 77, 015006 (2008)

34. D.A. Demir, M. Frank, K. Huitu, S.K. Rai, I. Turan, Phys. Rev. D 78, 035013 (2008)

35. D.A. Demir, M. Frank, D.K. Ghosh, K. Huitu, S.K. Rai, I. Turan, Phys. Rev. D 79, 095006 (2009)

36. R.M. Francis, M. Frank, C.S. Kalman, Phys. Rev. D 43, 2369 (1991)

37. K. Huitu, J. Maalampi, M. Raidal, Phys. Lett. B 328, 60 (1994)

38. K. Huitu, J. Maalampi, M. Raidal, Nucl. Phys. B 420, 449 (1994)

39. G. Hinshaw et al. [WMAP Collaboration], Astrophys. J. Suppl. 208, 19 (2013)

40. P.A.R. Ade et al. [Planck Collaboration], Astron. Astrophys. 594, A13 (2016)

41. D.A. Demir, M. Frank, I. Turan, Phys. Rev. D 73, 115001 (2006)

42. A. Chatterjee, M. Frank, B. Fuks, K. Huitu, S. Mondal, S.K. Rai, H. Waltari, Phys. Rev. D 99, 035017 (2019)

43. M. Frank, B. Fuks, K. Huitu, S.K. Rai, H. Waltari, JHEP 1705, 015 (2017)

44. A. Alloul, M. Frank, B. Fuks, M. Rausch de Traubenberg, JHEP 1310, 033 (2013)

45. M. Frank, B. Korutlu, Phys. Rev. D 83, 073007 (2011) 
46. W. Porod, F. Staub, Comput. Phys. Commun. 183, 2458 (2012)

47. F. Staub, Comput. Phys. Commun. 185, 1773 (2014)

48. V. Khachatryan et al. [CMS Collaboration], JHEP 1410, 160 (2014)

49. M. Aaboud et al. [ATLAS Collaboration], arXiv:1608.00890 [hep-ex]

50. K.S. Babu, X.G. He, E. Ma, Phys. Rev. D 36, 878 (1987)

51. T. Saito et al. [Belle Collaboration], Phys. Rev. D 91, 052004 (2015)

52. T. Hermann, M. Misiak, M. Steinhauser, JHEP 1211, 036 (2012)

53. S. Chatrchyan et al. [CMS Collaboration], Eur. Phys. J. C 72, 2189 (2012)

54. CMS Collaboration [CMS Collaboration], CMS-PAS-HIG-14-039 (2016)

55. CMS Collaboration [CMS Collaboration], CMS-PAS-HIG-16-036 (2017)

56. K.S. Babu, A. Patra, Phys. Rev. D 93, 055030 (2016)

57. M. Aaboud et al. [ATLAS Collaboration], Phys. Rev. D 96, 052004 (2017)

58. A.M. Sirunyan et al. [CMS Collaboration], Phys. Lett. B 769, 520 (2017) [Erratum: Phys. Lett. B 772, 882 (2017)]

59. W.Y. Keung, G. Senjanovic, Phys. Rev. Lett. 50, 1427 (1983)

60. V. Khachatryan et al. [CMS Collaboration], Eur. Phys. J. C 74, 3149 (2014)

61. P. Achard et al. [L3 Collaboration], Phys. Lett. B 517, 75 (2001)

62. U. Egede, M. Wielers, M. Peterson, ATL-PHYS-97-097

63. U. Egede, M. Wielers, M. Peterson, ATL-GE-PN-97

64. U. Egede, M. Wielers, M. Peterson, CERN-ATLPHYS- 97-097

65. E. Aprile et al. [XENON Collaboration], Phys. Rev. Lett. 121, 111302 (2018)

66. X. Cui et al. [PandaX-II Collaboration], Phys. Rev. Lett. 119, 181302 (2017)

67. M. Ackermann et al. [Fermi-LAT Collaboration], Phys. Rev. Lett. 115, 231301 (2015)

68. M. Backovic, A. Martini, O. Mattelaer, K. Kong, G. Mohlabeng, Phys. Dark Univ. 9-10, 37 (2015)

69. G. Belanger, F. Boudjema, A. Pukhov, A. Semenov, Comput. Phys. Commun. 192, 322 (2015)

70. J. Edsjo, P. Gondolo, Phys. Rev. D 56, 1879 (1997)

71. S. Profumo, A. Provenza, JCAP 0612, 019 (2006)

72. K.S. Babu, C.F. Kolda, Phys. Rev. Lett. 84, 228 (2000)

73. D.S. Akerib et al. [LUX Collaboration], Phys. Rev. Lett. 118, 021303 (2017)

74. M. Aguilar et al. [AMS Collaboration], Phys. Rev. Lett. 117, 091103 (2016)

75. J.A. Grifols, A. Mendez, G.A. Schuler, Mod. Phys. Lett. A 4, 1485 (1989)

76. J.F. Gunion, J. Grifols, A. Mendez, B. Kayser, F.I. Olness, Phys. Rev. D 40, 1546 (1989)

77. K. Huitu, J. Maalampi, A. Pietila, M. Raidal, Nucl. Phys. B 487, 27 (1997)

78. M. Aaboud et al. [ATLAS Collaboration], Eur. Phys. J. C 78, 199 (2018)

79. K.S. Babu, A. Patra, S.K. Rai, Phys. Rev. D 88, 055006 (2013)

80. S.K. Gupta, B. Mukhopadhyaya, S.K. Rai, Phys. Rev. D 75, 075007 (2007) 